\title{
Specifying Transaction Control to Serialize Concurrent Program Executions ${ }^{\star \dagger}$
}

\author{
Egon Börger ${ }^{1}$ and Klaus-Dieter Schewe ${ }^{2}$ \\ 1 Università di Pisa, Dipartimento di Informatica, I-56125 Pisa, Italy boerger@di.unipi.it \\ 2 Software Competence Centre Hagenberg, A-4232 Hagenberg, Austria klaus-dieter.schewe@scch.at
}

\begin{abstract}
We define a programming language independent transaction controller and an operator which when applied to concurrent programs with shared locations turns their behavior with respect to some abstract termination criterion into a transactional behavior. We prove the correctness property that concurrent runs under the transaction controller are serialisable. We specify the transaction controller TACTL and the operator TA in terms of Abstract State Machines. This makes TACTL applicable to a wide range of programs and in particular provides the possibility to use it as a plug-in when specifying concurrent system components in terms of Abstract State Machines.
\end{abstract}

\section{Introduction}

This paper is about the use of transactions as a common means to control concurrent access of programs to shared locations and to avoid that values stored at these locations are changed almost randomly. A transaction controller interacts with concurrently running programs (read: sequential components of an asynchronous system) to control whether access to a shared location can be granted or not, thus ensuring a certain form of consistency for these locations. A commonly accepted consistency criterion is that the joint behavior of all transactions (read: programs running under transactional control) with respect to the shared locations is equivalent to a serial execution of those programs. Serialisability guarantees that each transaction can be specified independently from the transaction controller, as if it had exclusive access to the shared locations.

It is expensive and cumbersome to specify transactional behavior and prove its correctness again and again for components of the great number of concurrent systems. Our goal is to define once and for all an abstract (i.e. programming language independent) transaction controller TACTL which can simply be "plugged in" to turn the behavior of concurrent programs (read: components $M$ of any given asynchronous system $\mathcal{M}$ ) into a transactional one. This involves to also define an operator $T A(M, \mathrm{TACTL})$ which forces the programs $M$ to listen to the controller TACTL when trying to access shared locations.

For the sake of generality we define the operator and the controller in terms of Abstract State Machines (ASMs) which can be read and understood as pseudo-code so that TACTL and the operator $T A$ can be applied to code written in any programming language (to be precise: whose programs come with a notion of single step, the level where our controller imposes shared memory access constraints to guarantee transactional code behavior). On the other side, the precise semantics underlying ASMs (for which we refer the reader to [5]) allows us to mathematically prove the correctness of our controller and operator.

\footnotetext{
* The research reported in this paper results from the project Behavioural Theory and Logics for Distributed Adaptive Systems supported by the Austrian Science Fund (FWF): [P26452-N15].

$\dagger$ The final publication is available at Springer via https://doi.org/10.1007/978-3-662-43652-3_13.
} 
We concentrate here on transaction controllers that employ locking strategies such as the common two-phase locking protocol (2PL). That is, each transaction first has to acquire a (read- or write-) lock for a shared location, before the access is granted. Locks are released after the transaction has successfully committed and no more access to the shared locations is necessary. There are of course other approaches to transaction handling, see e.g. 6]14]15]17] and the extensive literature there covering classical transaction control for flat transactions, timestamp-based, optimistic and hybrid transaction control protocols, as well as non-flat transaction models such as sagas and multi-level transactions.

We define TACTL and the operator TA in Sect. 2 and the TACTL components in Sect. 3 . In Sect. 4 we prove the correctness of these definitions.

\section{The Transaction Operator $T A(M, \mathrm{TACTL})$}

As explained above, a transaction controller performs the lock handling, the deadlock detection and handling, the recovery mechanism (for partial recovery) and the commit of single machines. Thus we define it as consisting of four components specified in Sect. 3.

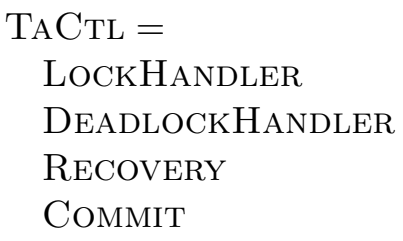

The operator $T A(M, \mathrm{TACTL})$ transforms the components $M$ of any concurrent system (asynchronous ASM) $\mathcal{M}=\left(M_{i}\right)_{i \in I}$ into components of a concurrent system $T A(\mathcal{M}$, TACTL) where each $T A\left(M_{i}, \mathrm{TACTL}_{\mathrm{T}}\right)$ runs as transaction under the control of TACTL:

$$
T A(\mathcal{M}, \mathrm{TACTL})=\left(\left(T A\left(M_{i}, \mathrm{TACTL}\right)\right)_{i \in I}, \mathrm{TACTL}\right)
$$

TACTL keeps a dynamic set TransAct of those machines $M$ whose runs it currently has to supervise to perform in a transactional manner until $M$ has Terminated its transactional behavior (so that it can Commit it) ${ }^{3}$ To turn the behavior of a machine $M$ into a transactional one, first of all $M$ has to register itself with the controller TACTL, read: to be inserted into the set of currently to be handled TransActions. To Undo as part of a recovery some steps $M$ made already during the given transactional run segment of $M$, a last-in first-out queue history $(M)$ is needed which keeps track of the states the transactional run goes through; when $M$ enters the set TransAct the history $(M)$ has to be initialized (to the empty queue).

The crucial transactional feature is that each non private (i.e. shared or monitored or output) location $l$ a machine $M$ needs to read or write for performing a step has to be LockedBy $(M)$ for this purpose; $M$ tries to obtain such locks by calling the LOCKHANDLER. In case no newLocks are needed by $M$ in its currState or the needed newLocks can be Granted by the LockHANDLER, $M$ performs its next step; in addition, for a possible future recovery, the machine has to RECORD in its history $(M)$ the current values of those locations which are (possibly over-) written by this $M$-step together with the obtained newLocks. Then $M$ continues its transactional behavior until it is Terminated. In case the needed newLocks are Refused, namely because another machine $N$ in TransAct for some needed $l$ has $W$-Locked $(l, N)$ or (in

\footnotetext{
${ }^{3}$ In this paper we deliberately keep the termination criterion abstract so that it can be refined in different ways for different transaction instances.
} 
case $M$ wants a W-(rite)Lock) has $R$-Locked $(l, N), M$ has to Wait for $N$; in fact it continues its transactional behavior by calling again the LOCKHANDLER for the needed newLocksuntil the needed locked locations are unlocked when $N$ 's transactional behavior is COMmiTed, whereafter a new request for these locks this time may be Granted to $M \square$

As a consequence deadlocks may occur, namely when a cycle occurs in the transitive closure Wait* of the Wait relation. To resolve such deadlocks the DEADLOCKHANDLER component of TACTL chooses some machines as Victims for a recovery 5 After a victimized machine $M$ is Recovered by the RECOVERY component of TACTL, so that $M$ can exit its waitForRecovery state, it continues its transactional behavior.

This explains the following definition of $T A(M, \mathrm{TACTL})$ as a control state ASM, i.e. an ASM with a top level Finite State Machine control structure. We formulate it by the flowchart diagram of Fig. 1, which has a precise control state ASM semantics (see the definition in [5, Ch.2.2.6]). The components for the recovery feature are highlighted in the flowchart by a colouring that differs from that of the other components. The macros which appear in Fig. 1 and the components of TACTL are defined below.

The predicate NewLocksNeededBy $(M)$ holds if in the current state of $M$ at least one of two cases happens: ${ }^{6}$ either $M$ to perform its step in this state reads some shared or monitored location which is not yet LockedBy $(M)$ or $M$ writes some shared or output location which is not yet $\operatorname{LockedBy}(M)$ for writing. A location can be $\operatorname{LockedBy}(M)$ for reading $(R$-Locked $(l, M))$ or for writing $(W$-Locked $(l, M))$. Formally:



The overWrittenValues are the currState $(M)$-values (retrieved by the eval-function) of those shared or output locations ( $f$, args) which are written by $M$ in its currState $(M)$. To RECORD the set of these values together with the obtained newLocks means to append the

\footnotetext{
${ }^{4}$ As suggested by a reviewer, a refinement (in fact a desirable optimization) consists in replacing such a waiting cycle by suspending $M$ until the needed locks are released. Such a refinement can be obtained in various ways, a simple one consisting in letting $M$ simply stay in waitForLocks until the newLocks CanBeGranted and refining LOCKHANDLER to only choose pairs $(M, L) \in$ LockRequest where it can GRANTREQUeSTEdLocks $(M, L)$ and doing nothing otherwise (i.e. defining $\operatorname{RefuseReQuestedLocks}(M, L)=$ skip). See Sect. 3

${ }^{5}$ To simplify the serializability proof in Sect 3 and without loss of generality we define a reaction of machines $M$ to their victimization only when they are in $\operatorname{ctl} \_$state $(M)=$ TA-ctl (not in $c t l_{-}$state $(M)=$ waitForLocks). This is to guarantee that no locks are Granted to a machine as long as it does waitForRecovery.

${ }^{6}$ See [5, Ch.2.2.3] for the classification of locations and functions.

7 For layout reasons we omit in Fig 1 the arguments of the functions newLocks and over Written Val.

${ }^{8}$ By the second argument currState $(M)$ of newLocks (and below of overWritten Val) we indicate that this function of $M$ is a dynamic function which is evaluated in each state of $M$, namely by computing in this state the sets $\operatorname{ReadLoc}(M)$ and WriteLoc $(M)$; see Sect. 4 for the detailed definition.

${ }^{9}$ By $\bar{X}$ we denote the complement of $X$.
} 


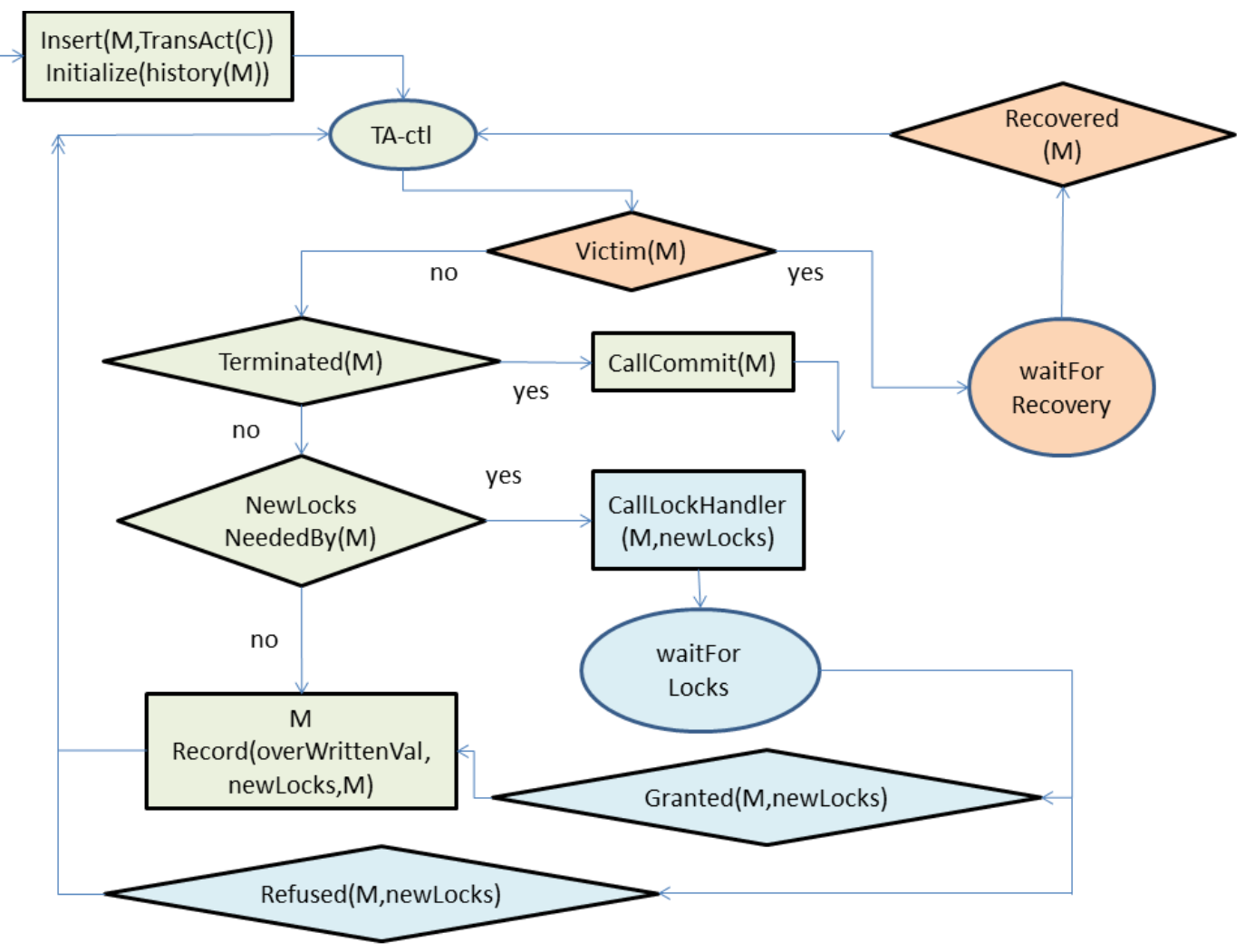

Fig. 1. TA(M,C)

pair of these two sets to the history queue of $M$ from where upon recovery the values and the locks can be retrieved.

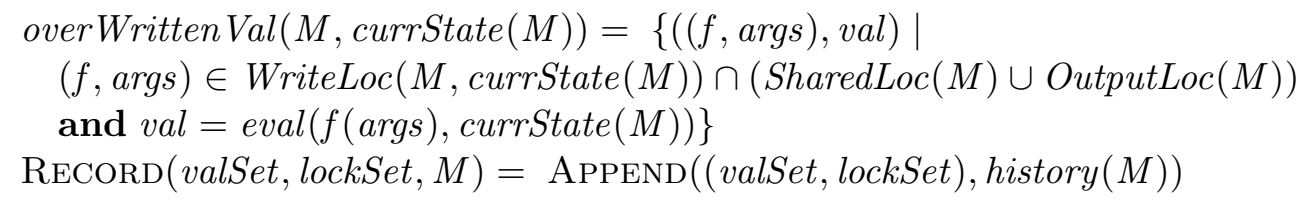

To CallLockHandler for the newLocks requested by $M$ in its currState $(M)$ means to $\operatorname{InSERT}(M$, newLocks $)$ into the LOCKHANDLER's set of to be handled LockRequests. Similarly we let CallCommit(M) stand for insertion of $M$ into a set CommitRequest of the Commit component.

$\operatorname{CalllockHandler}(M, L)=\operatorname{Insert}((M, L)$, LockRequest $)$

$\operatorname{CallCommit}(M)=\operatorname{Insert}(M$, CommitRequest $)$

\section{The Transaction Controller Components}

A CallCommit(M) by machine $M$ enables the Commit component. Using the choose operator we leave the order in which the CommitRequests are handled refinable by different instantiations of TACTL. 
Commiting $M$ means to UnLOCK all locations $l$ that are LockedBy $(M)$. Note that each lock obtained by $M$ remains with $M$ until the end of $M$ 's transactional behavior. Since $M$ performs a CALLCOMmiT(M) when it has Terminated its transactional computation, nothing more has to be done to Commit $M$ besides deleting $M$ from the sets of CommitRequests and still to be handled TransActions 10

Note that the locations $R$-Locked $(l, M)$ and $W$-Locked $(l, M)$ are shared by the Commit, LOCKHANDLER and RECOVERY components, but these components never have the same $M$ simultaneously in their request resp. Victim set since when machine $M$ has performed a CallCommit(M), it has Terminated its transactional computation and does not participate any more in any $(M, L) \in$ LockRequest or Victimization.

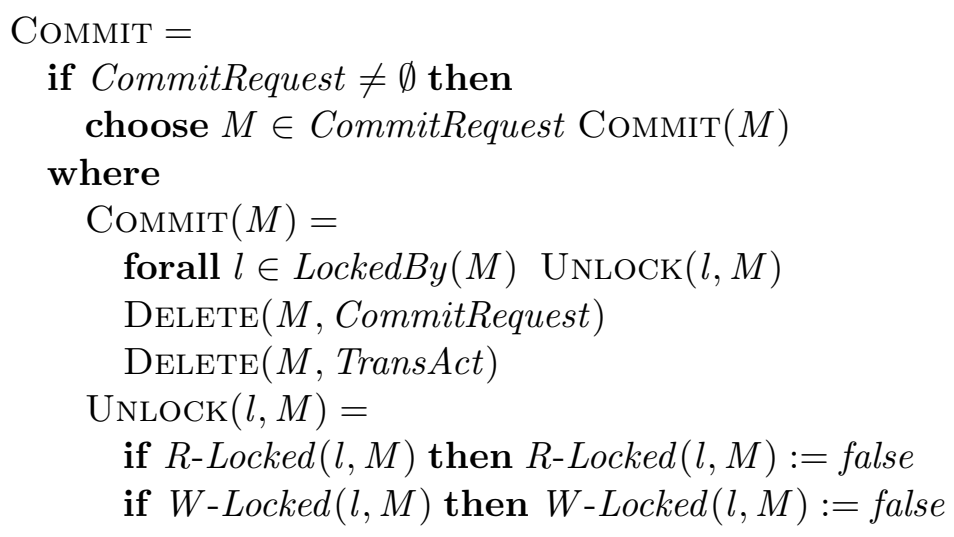

As for COMmit also for the LOCKHANDLER we use the choose operator to leave the order in which the LockRequests are handled refinable by different instantiations of TACTL.

The strategy we adopt for lock handling is to refuse all locks for locations requested by $M$ if at least one of the following two cases happens:

- some of the requested locations is $W$-Locked by another transactional machine $N \in$ TransAct,

- some of the requested locations is a WriteLocation that is $R$-Locked by another transactional machine $N \in$ TransAct.

This definition implies that multiple transactions may simultaneoulsy have a $R$-Lock on some location. It is specified below by the predicate CannotBeGranted.

To RefuseRequestedLocks it suffices to set the communication interface Refused of $T A(M, \mathrm{TACTL})$; this makes $M W a i t$ for each location $l$ that is $W$-Locked $(l, N)$ and for each WriteLocation that is $R$-Locked $(l, N)$ by some other transactional component machine $N \in$ TransAct.

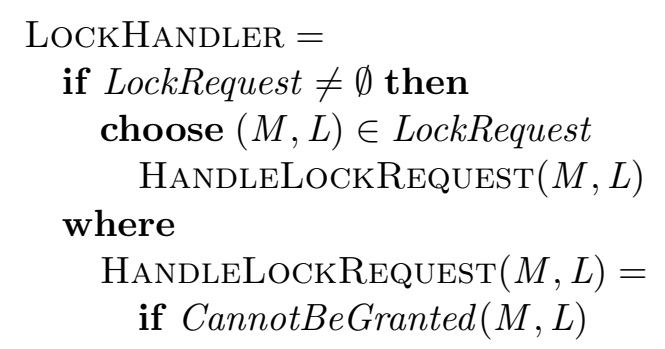

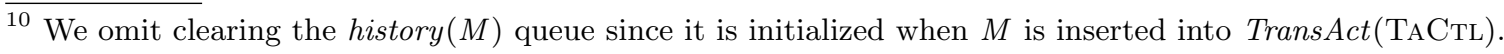




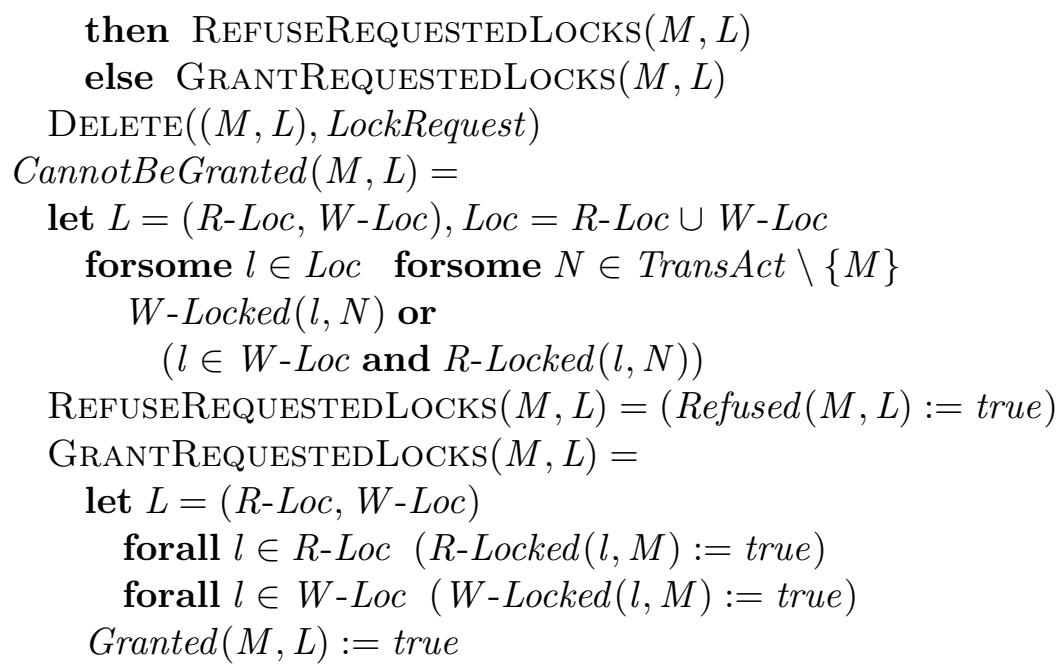

A Deadlock originates if two machines are in a Wait cycle, otherwise stated if for some (not yet Victimized) machine $M$ the pair $(M, M)$ is in the transitive (not reflexive) closure Wait* of Wait. In this case the DEADLOCKHANDLER selects for recovery a (typically minimal) subset of Deadlocked transactions toResolve - they are Victimized to waitForRecovery, in which mode (control state) they are backtracked until they become Recovered. The selection criteria are intrinsically specific for particular transaction controllers, driving a usually rather complex selection algorithm in terms of number of conflict partners, priorities, waiting time, etc. In this paper we leave their specification for TACTL abstract (read: refinable in different directions) by using the choose operator.

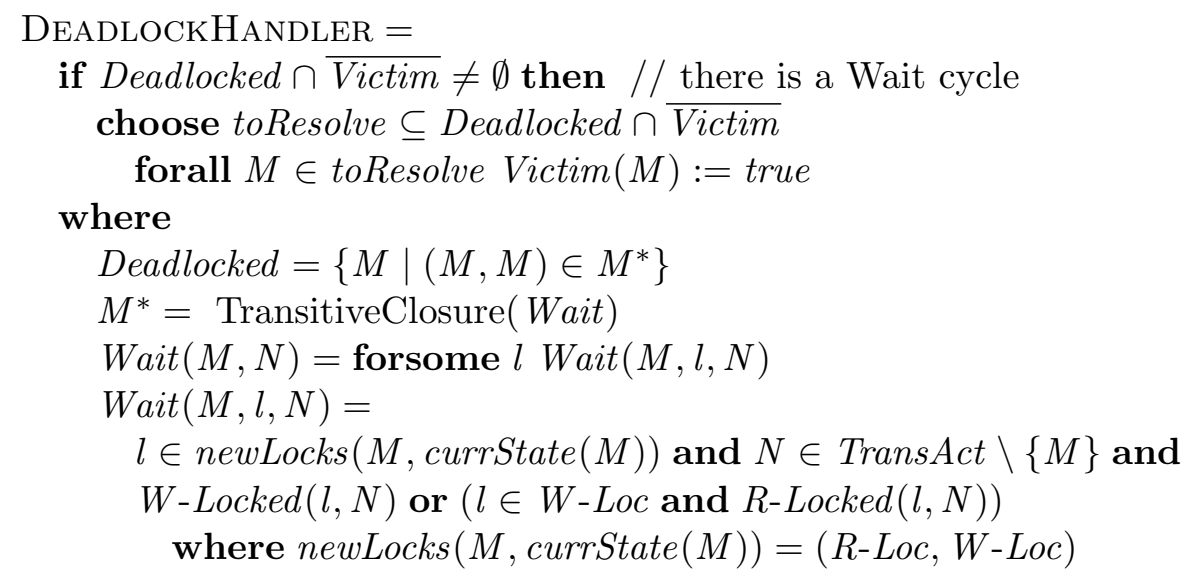

Also for the RECOVERY component we use the choose operator to leave the order in which the Victims are chosen for recovery refinable by different instantiations of TACTL. To be Recovered a machine $M$ is backtracked by $\operatorname{Undo}(M)$ steps until $M$ is not Deadlocked any more, in which case it is deleted from the set of Victims, so that be definition it is Recovered. This happens at the latest when history $(M)$ has become empty.






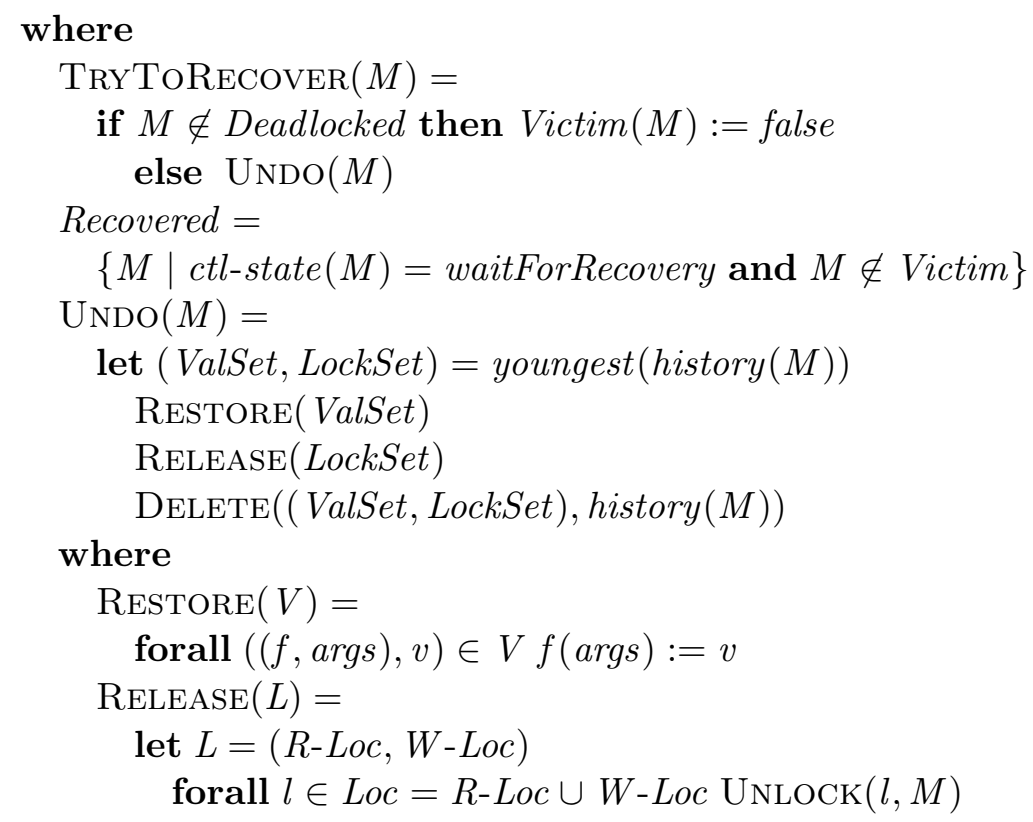

Note that in our description of the DeadlockHandler and the (partial) Recovery we deliberately left the strategy for victim seclection and UNDO abstract leaving fairness considerations to be discussed elsewhere. It is clear that if always the same victim is selected for partial recovery, the same deadlocks may be created again and again. However, it is well known that fairness can be achieved by choosing an appropriate victim selection strategy.

\section{Correctness Theorem}

In this section we show the desired correctness property: if all monitored or shared locations of any $M_{i}$ are output or controlled locations of some other $M_{j}$ and all output locations of any $M_{i}$ are monitored or shared locations of some other $M_{j}$ (closed system assumption) ${ }^{11}$, each run of $T A(\mathcal{M}, \mathrm{TACTL})$ is equivalent to a serialization of the terminating $M_{i}$-runs, namely the $M_{i_{1}}$-run followed by the $M_{i_{2}}$-run etc., where $M_{i_{j}}$ is the $j$-th machine of $\mathcal{M}$ which performs a commit in the $T A\left(\mathcal{M}, \mathrm{TACTL}_{\mathrm{T}}\right)$ run. To simplify the exposition (i.e. the formulation of statement and proof of the theorem) we only consider machine steps which take place under the transaction control, in other words we abstract from any step $M_{i}$ makes before being InsERTed into or after being DeLETed from the set TransAct of machines which currently run under the control of TACTL.

First of all we have to make precise what a serial multi-agent ASM run is and what equivalence of $T A\left(\mathcal{M}, \mathrm{TACTL}_{\mathrm{T}}\right)$ runs means in the general multi-agent ASM framework.

Definition of run equivalence. Let $S_{0}, S_{1}, S_{2}, \ldots$ be a (finite or infinite) run of the system $T A\left(\mathcal{M}, \mathrm{TACTL}_{\mathrm{T}}\right)$. In general we may assume that TACTL runs forever, whereas each machine $M \in \mathcal{M}$ running as transaction will be terminated at some time - at least after commit $M$ will only change values of non-shared and non-output locations ${ }^{12}$. For $i=0,1,2, \ldots$ let $\Delta_{i}$

11 This assumption means that the environment is assumed to be one of the component machines.

${ }^{12}$ It is possible that one ASM $M$ enters several times as a transaction controlled by TACTL. However, in this case each of these registrations will be counted as a separate transaction, i.e. as different ASMs in $\mathcal{M}$. 
denote the unique, consistent update set defining the transition from $S_{i}$ to $S_{i+1}$. By definition of $T A\left(\mathcal{M}, \mathrm{TACTL}_{\mathrm{T}}\right)$ the update set is the union of the update sets of the agents executing $M \in \mathcal{M}$ resp. TACTL:

$$
\Delta_{i}=\bigcup_{M \in \mathcal{M}} \Delta_{i}(M) \cup \Delta_{i}(\mathrm{TACTL}) .
$$

$\Delta_{i}(M)$ contains the updates defined by the ASM $T A\left(M\right.$, TACTL) in state $S_{2}^{13}$ and $\Delta_{i}$ (TACTL) contains the updates by the transaction controller in this state. The sequence of update sets $\Delta_{0}(M), \Delta_{1}(M), \Delta_{2}(M), \ldots$ will be called the schedule of $M$ (for the given transactional run).

To generalise for transactional ASM runs the equivalence of transaction schedules known from database systems [6, p.621ff.] we now define two cleansing operations for ASM schedules. By the first one (i) we eliminate all (in particular unsuccessful-lock-request) computation segments which are without proper $M$-updates; by the second one (ii) we eliminate all $M$ steps which are related to a later $\operatorname{Undo}(M)$ step by the RECOVERY component:

(i) Delete from the schedule of $M$ each $\Delta_{i}(M)$ where one of the following two properties holds:

- $\Delta_{i}(M)=\emptyset\left(M\right.$ contributes no update to $\left.S_{i}\right)$,

- $\Delta_{i}(M)$ belongs to a step of an $M$-computation segment where $M$ in its ctl_state $(M)=$ TA-ctl does CallLockHandler $(M, n e w L o c k s)$ and in its next step moves from controlstate waitForLocks back to control state TA-ctl, because the LOCKHANDLER refused new locks by Refused $(M$, newLocks $), 14$

In such computation steps $M$ makes no proper update.

(ii) Repeat choosing from the schedule of $M$ a pair $\Delta_{j}(M)$ with later $\Delta_{j^{\prime}}(M)\left(j<j^{\prime}\right)$ which belong to the first resp. second of two consecutive $M$-Recovery steps defined as follows:

- a (say $M$-RecoveryEntry) step whereby $M$ in state $S_{j}$ moves from control-state TA-ctl to waitForRecovery, because it became a Victim,

- the next $M$-step (say $M$-RecoveryExit) whereby $M$ in state $S_{j^{\prime}}$ moves back to control state TA-ctl because it has been Recovered.

In these two $M$-Recovery steps $M$ makes no proper update. Delete:

(a) $\Delta_{j}(M)$ and $\Delta_{j^{\prime}}(M)$,

(b) the $(($ Victim, $M)$, true $)$ update from the corresponding $\Delta_{t}$ (TACTL) $(t<j)$ which in state $S_{j}$ triggered the $M$-RecoveryEntry,

(c) TryToRecover $(M)$-updates in any update set $\Delta_{i+k}$ (TACTL) between the considered $M$-RecoveryEntry and $M$-RecoveryExit step $\left(i<j<i+k<j^{\prime}\right)$,

(d) each $\Delta_{i^{\prime}}(M)$ belonging to the $M$-computation segment from TA-ctl back to TA-ctl which contains the proper $M$-step in $S_{i}$ that is UNDOne in $S_{i+k}$ by the considered $\operatorname{TryToReCOver}(M)$ step; besides control state and RECORD updates these $\Delta_{i^{\prime}}(M)$ contain updates $(\ell, v)$ with $\ell=\left(f,\left(v_{a l} l_{S_{i}}\left(t_{1}\right), \ldots, v a l_{S_{i}}\left(t_{n}\right)\right)\right)$ where the corresponding UNDO updates are $\left(\ell, \operatorname{val}_{S_{i}}\left(f\left(t_{1}, \ldots, t_{n}\right)\right)\right) \in \Delta_{i+k}(\mathrm{TACTL})$,

\footnotetext{
$\overline{13}$ We use the shorthand notation $\Delta_{i}(M)$ to denote $\Delta_{i}(T A(M$, TACTL $))$; in other words we speak about steps and updates of $M$ also when they really are done by $T A(M$, TACTL $)$. Mainly this is about transitions between the control states, namely TA-ctl, waitForLocks, waitForRecovery (see Fig 1), which are performed during the run of $M$ under the control of the transaction controller TACTL. When we want to name an original update of $M$ (not one of the updates of $c t l_{-}$state $(M)$ or of the RECORD component) we call it a proper $M$-update.

14 Note that by eliminating this CallLockHandler $(M, L)$ step also the corresponding LockHandler step HANDLELOCKREQUEST $(M, L)$ disappears in the run.
} 
(e) the HandleLockRequest $\left(M\right.$, newLocks)-updates in $\Delta_{l \prime}$ (TACTL) corresponding to $M$ 's CallLockHandler step (if any: in case newLocks are needed for the proper $M$-step in $\left.S_{i}\right)$ in state $S_{l}\left(l<l^{\prime}<i\right)$.

The sequence $\Delta_{i_{1}}(M), \Delta_{i_{2}}(M), \ldots$ with $i_{1}<i_{2}<\ldots$ resulting from the application of the two cleansing operations as long as possible - note that confluence is obvious, so the sequence is uniquely defined - will be called the cleansed schedule of $M$ (for the given run).

Before defining the equivalence of transactional ASM runs we remark that $T A(\mathcal{M}, \mathrm{TACTL})$ has indeed several runs, even for the same initial state $S_{0}$. This is due to the fact that a lot of non-determinism is involved in the definition of this ASM. First, the submachines of TACTL are non-deterministic:

- In case several machines $M, M^{\prime} \in \mathcal{M}$ request conflicting locks at the same time, the LOCKHANDLER can only grant the requested locks for one of these machines.

- Commit requests are executed in random order by the Commit submachine.

- The submachine DEADlockHandleR chooses a set of victims, and this selection has been deliberately left abstract.

- The RECOVERY submachine chooses in each step a victim $M$, for which the last step will be undone by restoring previous values at updated locations and releasing corresponding locks.

Second, the specification of $T A\left(\mathcal{M}, \mathrm{TACTL}_{\mathrm{T}}\right)$ leaves deliberately open, when a machine $M \in \mathcal{M}$ will be started, i.e., register as a transaction in TransAct to be controlled by TACTL. This is in line with the common view that transactions $M \in \mathcal{M}$ can register at any time to the transaction controller TACTL and will remain under its control until they commit.

Definition 1. Two runs $S_{0}, S_{1}, S_{2}, \ldots$ and $S_{0}^{\prime}, S_{1}^{\prime}, S_{2}^{\prime}, \ldots$ of $T A(\mathcal{M}, \mathrm{TACTL})$ are equivalent iff for each $M \in \mathcal{M}$ the cleansed schedules $\Delta_{i_{1}}(M), \Delta_{i_{2}}(M), \ldots$ and $\Delta_{j_{1}}^{\prime}(M), \Delta_{j_{2}}^{\prime}(M), \ldots$ for the two runs are the same and the read locations and the values read by $M$ in $S_{i_{k}}$ and $S_{j_{k}}^{\prime}$ are the same.

That is, we consider runs to be equivalent, if all transactions $M \in \mathcal{M}$ read the same locations and see there the same values and perform the same updates in the same order disregarding waiting times and updates that are undone.

Definition of serializability. Next we have to clarify our generalised notion of a serial run, for which we concentrate on committed transactions - transactions that have not yet committed can still undo their updates, so they must be left out of consideration 15 . We need a definition of the read- and write-locations of $M$ in a state $S$, i.e. $\operatorname{Read} \operatorname{Loc}(M, S)$ and $\operatorname{WriteLoc}(M, S)$ as used in the definition of newLocks $(M, S)$.

The definition of Read/WriteLoc depends on the locking level, whether locks are provided for variables, pages, blocks, etc. To provide a definite definition, in this paper we give the definition at the level of abstraction of the locations of the underlying class $\mathcal{M}$ of component machines (ASMs) M. Refining this definition (and that of newLocks) appropriately for other locking levels does not innvalidate the main result of this paper.

We define $\operatorname{ReadLoc}(M, S)=\operatorname{ReadLoc}(r, S)$, where $r$ is the defining rule of the ASM $M$, and analogously $\operatorname{WriteLoc}(M, S)=\operatorname{WriteLoc}(r, S)$. Then we use structural induction

\footnotetext{
$\overline{15}$ Alternatively, we could concentrate on complete, infinite runs, in which only committed transactions occur, as eventually every transaction will commit - provided that fairness can be achieved.
} 
according to the definition of ASM rules in [5, Table 2.2]. As an auxiliary concept we need to define inductively the read and write locations of terms and formulae. The definitions use an interpretation $I$ of free variables which we suppress notationally (unless otherwise stated) and assume to be given with (as environment of) the state $S$. This allows us to write $\operatorname{ReadLoc}(M, S)$, WriteLoc $(M, S)$ instead of $\operatorname{ReadLoc}(M, S, I), \operatorname{ReadLoc}(M, S, I)$ respectively.

Read/Write Locations of Terms and Formulae. For state $S$ let $I$ be the given interpretation of the variables which may occur freely (in given terms or formulae). We write $v_{a l}$ (construct) for the evaluation of construct (a term or a formula) in state $S$ (under the given interpretation $I$ of free variables).

$$
\begin{aligned}
& \operatorname{ReadLoc}(x, S)=\operatorname{WriteLoc}(x, S)=\emptyset \text { for variables } x \\
& \operatorname{ReadLoc}\left(f\left(t_{1}, \ldots, t_{n}\right), S\right)= \\
& \quad\left\{\left(f,\left(\operatorname{val}_{S}\left(t_{1}\right), \ldots, \operatorname{val}_{S}\left(t_{n}\right)\right)\right)\right\} \cup \bigcup_{1 \leq i \leq n} \operatorname{ReadLoc}_{\left(t_{i}, S\right)} \\
& \operatorname{WriteLoc}\left(f\left(t_{1}, \ldots, t_{n}\right), S\right)=\left\{\left(f,\left(\operatorname{val}_{S}\left(t_{1}\right), \ldots, \operatorname{val}_{S}\left(t_{n}\right)\right)\right)\right\}
\end{aligned}
$$

Note that logical variables are not locations: they cannot be written and their values are not stored in a location but in the given interpretation $I$ from where they can be retrieved.

We define $\operatorname{Write} \operatorname{Loc}(\alpha, S)=\emptyset$ for every formula $\alpha$ because formulae are not locations one could write into. ReadLoc $(\alpha, S)$ for atomic formulae $P\left(t_{1}, \ldots, t_{n}\right)$ has to be defined as for terms with $P$ playing the same role as a function symbol $f$. For propositional formulae one reads the locations of their subformulae. In the inductive step for quantified formulae $\operatorname{domain}(S)$ denotes the superuniverse of $S$ minus the Reserve set [5, Ch.2.4.4] and $I_{x}^{d}$ the extension (or modification) of $I$ where $x$ is interpreted by a domain element $d$.

$$
\begin{aligned}
& \operatorname{ReadLoc}\left(P\left(t_{1}, \ldots, t_{n}\right), S\right)= \\
& \quad\left\{\left(P,\left(\operatorname{val}_{S}\left(t_{1}\right), \ldots, \operatorname{val}_{S}\left(t_{n}\right)\right)\right)\right\} \cup \cup_{1 \leq i \leq n} \operatorname{ReadLoc}\left(t_{i}, S\right) \\
& \operatorname{ReadLoc}(\neg \alpha)=\operatorname{ReadLoc}(\alpha) \\
& \operatorname{ReadLoc}\left(\alpha_{1} \wedge \alpha_{2}\right)=\operatorname{ReadLoc}\left(\alpha_{1}\right) \cup \operatorname{ReadLoc}\left(\alpha_{2}\right) \\
& \operatorname{ReadLoc}(\forall x \alpha, S, I)=\bigcup_{d \in \operatorname{domain}(S)} \operatorname{ReadLoc}\left(\alpha, S, I_{x}^{d}\right)
\end{aligned}
$$

Note that the values of the logical variables are not read from a location but from the modified state environment function $I_{x}^{d}$.

\section{Read/Write Locations of ASM Rules.}

$$
\begin{aligned}
& \operatorname{ReadLoc}(\text { skip, } S)=\operatorname{WriteLoc}(\mathbf{s k i p}, S)=\emptyset \\
& \operatorname{ReadLoc}\left(t_{1}:=t_{2}, S\right)=\operatorname{ReadLoc}\left(t_{1}, S\right) \cup \operatorname{ReadLoc}\left(t_{2}, S\right) \\
& \operatorname{WriteLoc}\left(t_{1}:=t_{2}, S\right)=\operatorname{WriteLoc}\left(t_{1}, S\right) \\
& \operatorname{ReadLoc}\left(\text { if } \alpha \text { then } r_{1} \text { else } r_{2}, S\right)=
\end{aligned}
$$




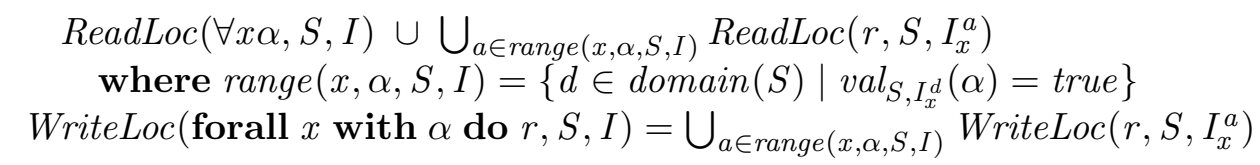

In the following cases the same scheme applies to read and write locations: ${ }^{16}$

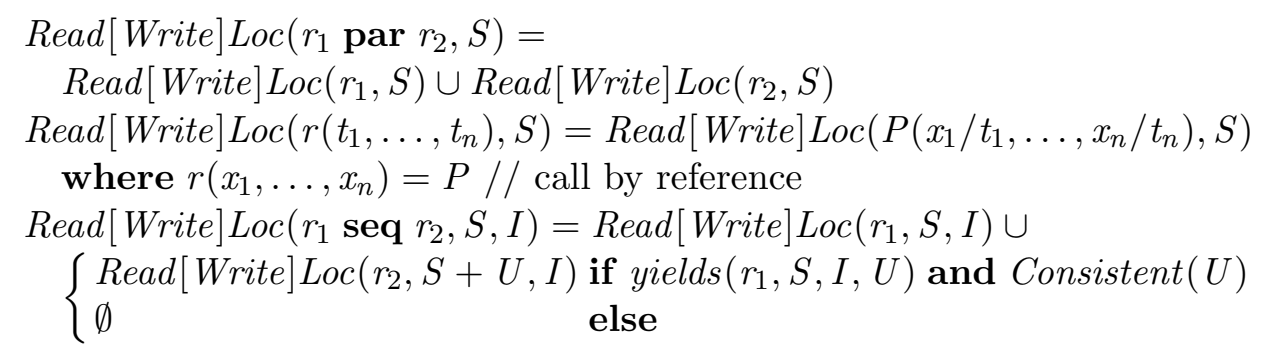

For choose rules we have to define the read and write locations simultaneously to guarantee that the same instance satisfying the selection condition is chosen for defining the read and write locations of the rule body $r$ :

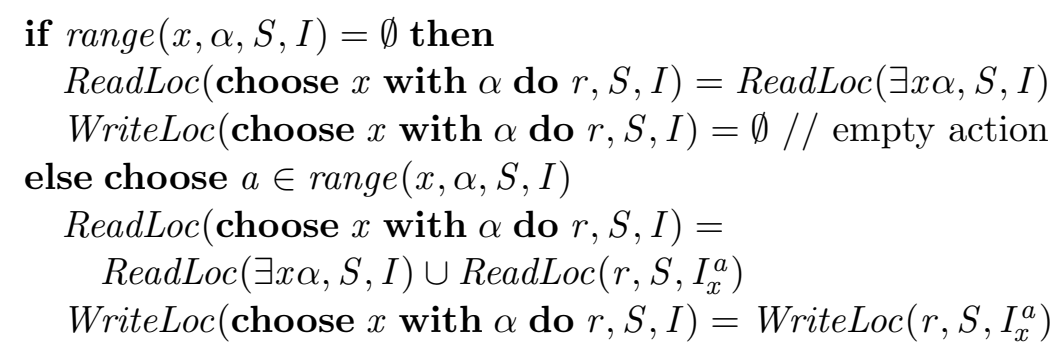

We say that $M$ has or is committed (in state $S_{i}$, denoted $\operatorname{Committed}\left(M, S_{i}\right)$ ) if step $\operatorname{Commit}(M)$ has been performed (in state $S_{i}$ ).

Definition 2. A run of $T A(\mathcal{M}, \mathrm{TACTL})$ is serial iff there is a total order $<$ on $\mathcal{M}$ such that the following two conditions are satisfied:

(i) If in a state $M$ has committed, but $M^{\prime}$ has not, then $M<M^{\prime}$ holds.

(ii) If $M$ has committed in state $S_{i}$ and $M<M^{\prime}$ holds, then the cleansed schedule $\Delta_{j_{1}}\left(M^{\prime}\right)$, $\Delta_{j_{2}}\left(M^{\prime}\right), \ldots$ of $M^{\prime}$ satisfies $i<j_{1}$.

That is, in a serial run all committed transactions are executed in a total order and are followed by the updates of transactions that did not yet commit.

Definition 3. A run of $T A\left(\mathcal{M}, \mathrm{TACTL}_{\mathrm{T}}\right)$ is serialisable iff it is equivalent to a serial run of $T A(\mathcal{M}, \mathrm{TACTL}) 17$

Theorem 1. Each run of $T A\left(\mathcal{M}, \mathrm{TACTL}_{\mathrm{T}}\right)$ is serialisable.

Proof. Let $S_{0}, S_{1}, S_{2}, \ldots$ be a run of $T A\left(\mathcal{M}, \mathrm{TACTL}_{\mathrm{T}}\right)$. To construct an equivalent serial run let $M_{1} \in \mathcal{M}$ be a machine that commits first in this run, i.e. $\operatorname{Committed}\left(M, S_{i}\right)$ holds for

$\left.\overline{{ }^{16} \text { In yields }\left(r_{1}\right.}, S, I, U\right) U$ denotes the update set produced by rule $r_{1}$ in state $S$ under $I$.

17 Modulo the fact that ASM steps permit simultaneous updates of multiple locations, this definition of serializability is equivalent to Lamport's sequential consistency concept [16]. 
some $i$ and whenever $\operatorname{Committed}\left(M, S_{j}\right)$ holds for some $M \in \mathcal{M}$, then $i \leq j$ holds. If there is more than one machine $M_{1}$ with this property, we randomly choose one of them.

Take the run of $T A\left(\left\{M_{1}\right\}\right.$, TACTL $)$ starting in state $S_{0}$, say $S_{0}, S_{1}^{\prime}, S_{2}^{\prime}, \ldots, S_{n}^{\prime}$. As $M_{1}$ commits, this run is finite. $M_{1}$ has been Deleted from TransAct and none of the TACTL components is triggered any more: neither COMMIT nor LOCKHANDLER because CommitRequest resp. LockRequest remain empty; not DEADLOCKHANDLER because Deadlock remains false since $M_{1}$ never Waits for any machine; not RECOVERY because Victim remains empty. Note that in this run the schedule for $M_{1}$ is already cleansed.

We now define a run $S_{0}^{\prime \prime}, S_{1}^{\prime \prime}, S_{2}^{\prime \prime}, \ldots$ (of $T A\left(\mathcal{M}-\left\{M_{1}\right\}\right.$, TACTL), as has to be shown) which starts in the final state $S_{n}^{\prime}=S_{0}^{\prime \prime}$ of the $T A\left(\left\{M_{1}\right\}\right.$, TACTL) run and where we remove from the run defined by the cleansed schedules $\Delta_{i}(M)$ for the originally given run all updates made by steps of $M_{1}$ and all updates in TACTL steps which concern $M_{1}$. Let

$$
\Delta_{i}^{\prime \prime}=\bigcup_{M \in \mathcal{M}-\left\{M_{1}\right\}} \Delta_{i}(M) \cup\left\{(\ell, v) \in \Delta_{i}(\mathrm{TACTL}) \mid(\ell, v) \text { does not concern } M_{1}\right\} .
$$

That is, in the update set $\Delta_{i}^{\prime \prime}$ all updates are removed from the original run which are done by $M_{1}$ - their effect is reflected already in the initial run segment from $S_{0}$ to $S_{n}^{\prime}$ - or are LOCKHANDLER updates involving a LockRequest $\left(M_{1}, L\right)$ or are $\operatorname{Victim}\left(M_{1}\right):=\operatorname{true}$ updates of the DeadlockHandler or are updates involving a $\operatorname{TrYToReCOVER}\left(M_{1}\right)$ step or are done by a step involving a $\operatorname{Commit}\left(M_{1}\right)$.

Lemma 1. $S_{0}^{\prime \prime}, S_{1}^{\prime \prime}, S_{2}^{\prime \prime}, \ldots$ is a run of $T A\left(\mathcal{M}-\left\{M_{1}\right\}\right.$, TACTL $)$.

Lemma 2. The run $S_{0}, S_{1}^{\prime}, S_{2}^{\prime}, \ldots, S_{n}^{\prime}, S_{1}^{\prime \prime}, S_{2}^{\prime \prime}, \ldots$ of $T A(\mathcal{M}, \mathrm{TACTL})$ is equivalent to the original run $S_{0}, S_{1}, S_{2}, \ldots$.

By induction hypothesis $S_{0}^{\prime \prime}, S_{1}^{\prime \prime}, S_{2}^{\prime \prime}, \ldots$ is serialisable, so $S_{0}, S_{1}^{\prime}, S_{2}^{\prime}, \ldots$ and thereby also $S_{0}, S_{1}, S_{2}, \ldots$ is serialisable with $M_{1}<M$ for all $M \in \mathcal{M}-\left\{M_{1}\right\}$.

Proof.(Lemma 1) We first show that omitting in $\Delta_{i}^{\prime \prime}$ every update from $\Delta_{i}$ (TACTL) which concerns $M_{1}$ does not affect updates by TACTL in $S_{i}^{\prime \prime}$ concerning $M \neq M_{1}$. In fact starting in the final $M_{1}$-state $S_{0}^{\prime \prime}, T A\left(\mathcal{M}-\left\{M_{1}\right\}\right.$, TACTL $)$ makes no move with a $\operatorname{Victim}\left(M_{1}\right):=$ true update and no move of Commit $\left(M_{1}\right)$ or HandleLockRequest $\left(M_{1}, L\right)$ or TrYToRecover $\left(M_{1}\right)$

It remains to show that every $M$-step defined by $\Delta_{i}^{\prime \prime}(M)$ is a possible $M$-step in a $T A(\mathcal{M}-$ $\left\{M_{1}\right\}$, TACTL) run starting in $S_{0}^{\prime \prime}$. Since the considered $M$-schedule $\Delta_{i}(M)$ is cleansed, we only have to consider any proper update step of $M$ in state $S_{i}^{\prime \prime}$ (together with its preceding lock request step, if any). If in $S_{i}^{\prime \prime} M$ uses newLocks, in the run by the cleansed schedules for the original run the locks must have been granted after the first COMMIT, which is done for $M_{1}$ before $S_{0}^{\prime \prime}$. Thus these locks are granted also in $S_{i}^{\prime \prime}$ as part of a $T A\left(\mathcal{M}-\left\{M_{1}\right\}\right.$, TACTL $)$ run step. If no newLocks are needed, that proper $M$-step depends only on steps computed after $S_{0}^{\prime \prime}$ and thus is part of a $T A\left(\mathcal{M}-\left\{M_{1}\right\}\right.$, TACTL $)$ run step.

Proof.(Lemma 2) The cleansed machine schedules in the two runs, the read locations and the values read there have to be shown to be the same. First consider any $M \neq M_{1}$. Since in the initial segment $S_{0}, S_{1}^{\prime}, S_{2}^{\prime}, \ldots, S_{n}^{\prime}$ no such $M$ makes any move so that its update sets in this computation segment are empty, in the cleansed schedule of $M$ for the run $S_{0}, S_{1}^{\prime}, S_{2}^{\prime}, \ldots, S_{n}^{\prime}, S_{1}^{\prime \prime}, S_{2}^{\prime \prime}, \ldots$ all these empty update sets disappear. Thus this cleansed schedule is the same as the cleansed schedule of $M$ for the run $S_{n}^{\prime}, S_{1}^{\prime \prime}, S_{2}^{\prime \prime}, \ldots$ and therefore by 
definition of $\Delta_{i}^{\prime \prime}(M)=\Delta_{i}(M)$ also for the original run $S_{0}, S_{1}, S_{2}, \ldots$ with same read locations and same values read there.

Now consider $M_{1}$, its schedule $\Delta_{0}\left(M_{1}\right), \Delta_{1}\left(M_{1}\right), \ldots$ for the run $S_{0}, S_{1}, S_{2}, \ldots$ and the corresponding cleansed schedule $\Delta_{i_{0}}\left(M_{1}\right), \Delta_{i_{1}}\left(M_{1}\right), \Delta_{i_{2}}\left(M_{1}\right), \ldots$ We proceed by induction on the cleansed schedule steps of $M_{1}$. When $M_{1}$ makes its first step using the $\Delta_{i_{0}}\left(M_{1}\right)$-updates, this can only be a proper $M_{1}$-step together with the corresponding RECORD updates (or a lock request directly preceding such a $\Delta_{i_{1}}\left(M_{1}\right)$-step) because in the computation with cleansed schedule each lock request of $M_{1}$ is granted and $M_{1}$ is not Victimized. The values $M_{1}$ reads or writes in this step (in private or locked locations) have not been affected by a preceding step of any $M \neq M_{1}$-otherwise $M$ would have locked before the non-private locations and keep the locks until it commits (since cleansed schedules are without UNDO steps), preventing $M_{1}$ from getting these locks which contradicts the fact that $M_{1}$ is the first machine to commit and thus the first one to get the locks. Therefore the values $M_{1}$ reads or writes in the step defined by $\Delta_{i_{0}}\left(M_{1}\right)$ (resp. also $\Delta_{i_{1}}\left(M_{1}\right)$ ) coincide with the corresponding location values in the first (resp. also second) step of $M_{1}$ following the cleansed schedule to pass from $S_{0}$ to $S_{1}^{\prime}$ (case without request of newLocks) resp. from $S_{0}$ to $S_{1}^{\prime}$ to $S_{2}^{\prime}$ (otherwise). The same argument applies in the inductive step which establishes the claim.

\section{Conclusion}

In this article we specified (in terms of Abstract State Machines) a transaction controller TACTL and a transaction operator which turn the behaviour of a set of concurrent programs into a transactional one under the control of TACTL. In this way the locations shared by the programs are accessed in a well-defined manner. For this we proved that all concurrent transactional runs are serialisable.

The relevance of the transaction operator is that it permits to concentrate on the specification of program behavior ignoring any problems resulting from the use of shared locations. That is, specifications can be written in a way that shared locations are treated as if they were exclusively used by a single program. This is valuable for numerous applications, as shared locations (in particular, locations in a database) are common, and random access to them is hardly ever permitted.

Furthermore, by shifting transaction control into the rigorous framework of Abstract State Machines we made several extensions to transaction control as known from the area of databases [6]. In the classical theory schedules are sequences containing read- and writeoperations of the transactions plus the corresponding read- and write-lock and commit events, i.e., only one such operation or event is treated at a time. In our case we exploited the inherent parallelism in ASM runs, so we always considered an arbitrary update set with usually many updates at the same time. Under these circumstances we generalised the notion of schedule and serialisability in terms of the synchronous parallelism of ASMs. In this way we stimulate also more parallelism in transactional systems.

Among further work we would like to be undertaken is to provide a (proven to be correct) implementation of our transaction controller and the $T A$ operator, in particular as plug-in for the CoreASM [87] or Asmeta [410] simulation engines. We would also like to see refinements or adaptations of our transaction controller model for different approaches to serialisability [14], see also the ASM-based treatment of multi-level transaction control in [15]. Last but not least we would like to see further detailings of our correctness proof to a mechanically 
verified one, e.g. using the ASM theories developed in KIV (see [1] for an extensive list of relevant publications) and PVS 91312] or the (Event-)B [23] theorem prover for an (Event-)B transformation of $T A\left(\mathcal{M}, \mathrm{TACTL}_{\mathrm{T}}\right)$ (as suggested in [1]).

Acknowledgement. We thank Andrea Canciani and some of our referees for useful comments to improve the paper.

\section{References}

1. The KIV system. http://www.informatik.uni-augsburg.de/lehrstuehle/swt/se/kiv/.

2. J.-R. Abrial. The B-Book. Cambridge University Press, Cambridge, 1996.

3. J.-R. Abrial. Modeling in Event-B. Cambridge University Press, 2010.

4. P. Arcaini, A. Gargantini, E. Riccobene, and P. Scandurra. A model-driven process for engineering a toolset for a formal method. Software, Practice and Experience, 41(2):155-166, 2011.

5. E. Börger and R. F. Stärk. Abstract State Machines. A Method for High-Level System Design and Analysis. Springer, 2003.

6. R. Elmasri and S. B. Navathe. Fundamentals of Database Systems. Addison Wesley, 2006.

7. R. Farahbod, V. Gervasi, and U. Glässer. CoreASM: An extensible ASM execution engine. Fundamenta Informaticae, 77(1-2):71-103, 2007.

8. R. Farahbod, V. Gervasi, and U. Glässer. Executable formal specifications of complex distributed systems with CoreASM. Science of Computer Programming, 79:23-38, 2014.

9. A. Gargantini and E. Riccobene. Encoding Abstract State Machines in PVS. In Y. Gurevich, P. Kutter, M. Odersky, and L. Thiele, editors, Abstract State Machines: Theory and Applications, volume 1912 of Lecture Notes in Computer Science, pages 303-322. Springer-Verlag, 2000.

10. A. Gargantini, E. Riccobene, and P. Scandurra. A metamodel-based language and a simulation engine for Abstract State Machines. Journal of Universal Computer Science, 14(12):1949-1983, 2008.

11. U. Glässer, S. Hallerstede, M. Leuschel, and E. Riccobene. Integration of Tools for Rigorous Software Construction and Analysis (Dagstuhl Seminar 13372). Dagstuhl Reports, 3(9):74-105, 2014.

12. W. Goerigk, A. Dold, T. Gaul, G. Goos, A. Heberle, F. W. von Henke, U. Hoffmann, H. Langmaack, H. Pfeifer, H. Ruess, and W. Zimmermann. Compiler correctness and implementation verification: The verifix approach. In P. Fritzson, editor, Int. Conf. on Compiler Construction, Proc. Poster Session of CC'96, Linköping, Sweden, 1996. IDA Technical Report LiTH-IDA-R-96-12.

13. G. Goos, H. von Henke, and H. Langmaack. Project verifix. http://www.info.uni-karlsruhe.de/ projects. php/id=28\&lang=en

14. J. Gray and A. Reuter. Transaction Processing: Concepts and Techniques. Morgan Kaufmann, 1993.

15. M. Kirchberg, K.-D. Schewe, and J. Zhao. Using Abstract State Machines for the design of multi-level transaction schedulers. In J.-R. Abrial and U. Glässer, editors, Rigorous Methods for Software Construction and Analysis - Papers Dedicated to Egon Börger on the Occasion of His 60th Birthday, volume 5115 of LNCS Festschrift, pages 65-77. Springer, 2009.

16. L. Lamport. How to make a multiprocessor computer that correctly executes multiprocess programs. IEEE Trans. Computers, 28(9):690-691, 1979.

17. K.-D. Schewe, T. Ripke, and S. Drechsler. Hybrid concurrency control and recovery for multi-level transactions. Acta Cybernetica, 14(3):419-453, 2000. 